\title{
Topological hypothesis on phase transitions: The simplest case
}

\author{
Ana C. Ribeiro Teixeira* and Daniel A. Stariolo ${ }^{\dagger}$ \\ Departamento de Física, Universidade Federal do Rio Grande do Sul, CP 15051, 91501-979 Porto Alegre, Brazil
}

(Received 26 March 2004; published 15 July 2004)

\begin{abstract}
We critically analyze the possibility of finding signatures of a phase transition by looking exclusively at static quantities of statistical systems, like, e.g., the topology of potential energy submanifolds (PES's). This topological hypothesis has been successfully tested in a few statistical models but up to now there has been no rigorous proof of its general validity. We make a new test of it analyzing the, probably, simplest example of a nontrivial system undergoing a continuous phase transition: the completely connected version of the spherical model. Going through the topological properties of its PES it is shown that, as expected, the phase transition is correlated with a change in their topology. Nevertheless, this change, as reflected in the behavior of a particular topological invariant, the Euler characteristic, is small, at variance with the strong singularity observed in other systems. Furthermore, it is shown that in the presence of an external field, when the phase transition is destroyed, a similar topology change in the submanifolds is still observed at the maximum value of the potential energy manifold, a level which nevertheless is thermodynamically inaccessible. This suggests that static properties of the PES's are not enough in order to decide whether a phase transition will take place; some input from dynamics seems necessary.
\end{abstract}

DOI: 10.1103/PhysRevE.70.016113

PACS number(s): 64.60.-i, 05.70.Fh, 02.40.Sf

\section{INTRODUCTION}

In a series of interesting papers [1-5] which appeared in the last few years the possibility has been advanced that phase transitions may be signaled by suitable changes in some topological properties of the configuration space manifold. This implies a different approach to phase transitions from the classical one based on singularities of thermodynamic potentials. The topological hypothesis implies a static point of view on phase transitions for it is based only on properties of static quantities, like the potential energy manifold. Under rather general conditions dynamics seems to play no role [7]. This is clearly a strong assumption and, if confirmed at least for a restricted class of systems, should provide a new and powerful approach to understand the microscopic mechanisms underlying a phase transition. Up to now the topological hypothesis has been verified in a few models, most notably the Hamiltonian $X Y$ mean field model $[4,8]$, the two-dimensional lattice $\phi^{4}$ model [3], the $k$-trigonometric model [5], and recently in the Bishop-Peyrard model of DNA denaturation [6]. The topology of these models was investigated by calculating a topological invariant, the Euler characteristic $\chi(v)$ defined on submanifolds $M_{v}$ of the potential energy manifold: $M_{v} \equiv\left\{q \in \mathcal{R}^{N} \mid V(q) \leqslant v\right\}$, where $V\left(q_{1}, \ldots, q_{N}\right)$ is the potential energy function of the system. In all the previous models the Euler characteristic shows a strong singularity at a critical level $v_{c}$ in correspondence with the critical values of the energy and temperature at the phase transition $e_{c}=T_{c} / 2+\left\langle v\left(T_{c}\right)\right\rangle\left[\left\langle v\left(T_{c}\right)\right\rangle=v_{c}\right]$. In the Hamiltonian $X Y$ mean-field model and in the $k$-trigonometric model, which is also a mean-field one, it was observed that $\lim _{N \rightarrow \infty}(1 / N) \ln \left|\chi_{v}\right|$ is singular at $v_{c}$. After this evidence the

\footnotetext{
*Electronic address: anacarol@if.ufrgs.br

†Electronic address: stariolo@if.ufrgs.br
}

question that remains to be answered refers to the necessary and sufficient conditions in order that a topology change of submanifolds of the configuration space reflects the presence of a phase transition. Recently a theorem was proved stating that a topology change of configuration space is in fact necessary $[9,10]$ for a phase transition to occur. The theorem covers a wide class of systems with smooth, finite range, and confining potentials bounded from below. But although at a phase transition a topology change must necessarily happen, the converse is not true. For it is known that topology changes are common in configuration space while they do not necessarily imply the presence of a phase transition. The next, more difficult task is to find sufficient conditions in order to relate topology changes with phase transitions. Up to now the only hints about what those conditions could be come from the observed behavior in the exactly solved $X Y$ and $k$-trigonometric models. Specially after the results in the $X Y$ model the authors conjectured that to entail a phase transition the topology change must involve the attachment of handles of $\mathcal{O}(N)$ different types on the same critical level [4]. We will show below a much simpler system undergoing a phase transition in which this mechanism is not present.

In this work we study the connection between the topology of the potential energy manifold and the thermodynamics of a very simple model: the completely connected ferromagnetic spherical model. The potential energy manifold of this system is a hypersphere and its topology is therefore trivial. We first discuss the model without an external field. The critical points of the energy function within the domain of the spherical constraint are two isolated symmetric minima corresponding to the ground states of the system and a highly degenerate maximum. There are no saddle points. The submanifolds $M_{v}$ at fixed $v$ correspond to two disconnected $(N-1)$-dimensional disks, joining each other and completing the whole sphere at the maximum level $v_{c}$. This level coincides with the critical value obtained from thermo- 
dynamics implying the coincidence between the topology change, the closing of the hypersphere, and the phase transition, in agreement with the topological hypothesis. Nevertheless, we will see that the Euler characteristic presents at best only a small discontinuity at the transition point and also only one handle is attached at the upper critical level which corresponds to the phase transition. This suggests that the sufficiency condition discussed above does not necessarily relate with the behavior of the Euler characteristic at the transition. In fact the topology change at the transition level in this model seems to be small in the sense that only one handle is attached in order to complete the whole manifold. Then we discuss the model in the presence of an external field. The topology is essentially the same as in the previous case, except that the symmetry between the two minima is broken. Now one minimum is the ground state and the other, a metastable state. We show that a topology change still exists where the hypersphere closes itself, but more importantly, this sector of the manifold is inaccessible to the physical system. As a consequence, this topology change (similar to the one occurring when there is no external field) cannot be related with a phase transition, which in fact is destroyed by the presence of a finite field. In order to conclude this we need to add some information from the whole problem - e.g., knowledge of the caloric curve, which depends on the dynamics. This suggests that topology alone may be not enough to decide whether a phase transition will take place for a given interaction potential.

\section{MODEL}

We studied the completely connected version of the classical spherical model introduced by Berlin and Kac [11]. It consists of a set of $N$ classical spin variables $\left\{s_{i} \in \mathcal{R}, i\right.$ $=1, \ldots, N\}$ which interact through the potential energy function,

$$
V=-\frac{J}{2 N} \sum_{i \neq j}^{N} s_{i} s_{j}-H \sum_{i}^{N} s_{i},
$$

and the spins are subject to a spherical constraint

$$
\sum_{i=1}^{N} s_{i}^{2}=N
$$

The exchange coupling $J>0$ corresponds to a ferromagnetic interaction, $H$ is an external field, and the factor $1 / N$ in the energy function is needed in order to make the model extensive in the thermodynamic limit.

The thermodynamics of the model can be computed exactly following closely the original solution of Berlin and Kac for the finite-dimensional version.

\section{A. Zero external field}

For $H=0$ a saddle point approach leads to a Curie-Weiss critical point at $\beta_{c} J=1$, where $\beta=1 / T$. The internal energy per particle behaves as

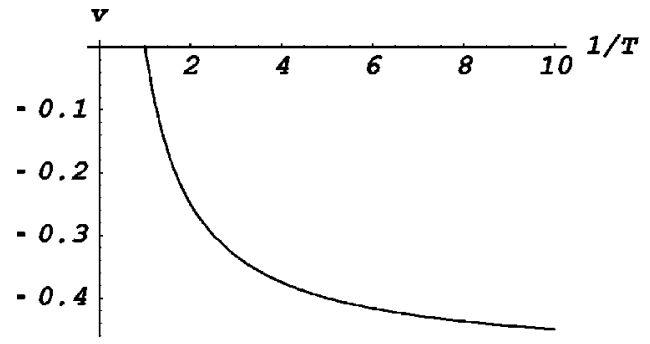

FIG. 1. Internal energy per particle as a function of inverse temperature in the completely connected spherical model for zero external field $(J=1)$.

$$
v= \begin{cases}\frac{1}{2 \beta}-\frac{J}{2}, & T<T_{c}, \\ 0, & T>T_{c} .\end{cases}
$$

Consequently at the critical point the mean potential energy is $v_{c} \equiv v\left(\beta_{c}\right)=0$ as shown in Fig. 1 .

This is the relevant information needed on the phase transition in this model in order to test the topological hypothesis. Below it is shown that the level $v_{c}$ corresponds to the maximum of the potential energy per particle and precisely at this level a topological change takes place in the submanifolds $M_{v}$.

\section{B. Finite external field}

The saddle point approach when $H \neq 0$ leads to a saddle point equation which has a finite solution for any finite temperature. Consequently the phase transition is destroyed by the field [11]. In the thermodynamic limit the internal energy per particle is given by

$$
v=\frac{1}{2 \beta}-J z_{s}-\frac{H^{2}}{4 J\left(z_{s}-1 / 2\right)},
$$

where $z_{s}$ is the solution of the saddle point equation:

$$
\frac{1}{z_{s}}+\frac{\beta H^{2}}{2 J\left(z_{s}-1 / 2\right)^{2}}-2 \beta J=0 .
$$

A plot of the internal energy $v$ as a function of $\beta=1 / T$ is shown in Fig. 2. When $\beta \rightarrow \infty, v \rightarrow-3 / 2$, which is the energy of the ground state for $H=1$. For $\beta \rightarrow 0, v \rightarrow 0$, a value below the maximum of the potential energy per particle which is $v_{\max }=H^{2} / 2=1 / 2$ in this case. The conclusion is that the sys-

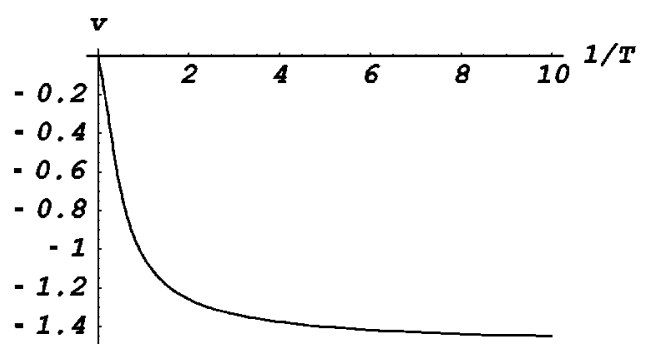

FIG. 2. Internal energy per particle as a function of inverse temperature for an external field $H=1(J=1)$. 
tem is unable to reach the levels of potential energy above $v=0$, for arbitrarily growing temperature. This is connected with the fact that the phase transition is absent when $H$ is finite. This behavior is reflected in the topology of the accessible submanifolds of potential energy, as will be seen below.

\section{CRITICAL POINTS AND TOPOLOGY OF THE POTENTIAL ENERGY MANIFOLD}

\section{A. Zero external field}

From the previous definition of the model it is clear that the whole energy manifold is a hypersphere in $N$ dimensions or, in topological language, an $(N-1)$-sphere. At a given level of potential energy $v$ the accessible submanifold is represented by the intersection of the energy surface (1) with the hypersphere. The topology of the energy function is easily revealed by diagonalizing the quadratic form. We can write [11]

$$
\sum_{i, j} s_{i} s_{j}=\mathbf{s}^{T} \mathbf{M s}
$$

with $\mathbf{M}$ the symmetric matrix with all elements equal to 1 except for the diagonal ones which are zero. This matrix is symmetric and is therefore diagonalized by means of an orthogonal transformation $\mathbf{V}$ such that

$$
\mathbf{M V} \mathbf{V}_{k}=\lambda_{k} \mathbf{V}_{k}, \quad \mathbf{V}^{T} \mathbf{V}=\mathbf{V}^{-1} \mathbf{V}=\mathcal{I},
$$

where $\mathbf{V}_{k}$ is the $k$ column of the transformation matrix.

Applying this transformation to $\mathbf{M}$ :

$$
\mathbf{s}^{T} \mathbf{M s}=\sum_{i=1}^{N} \lambda_{i} y_{i}^{2},
$$

where $\mathbf{y}=\mathbf{V}^{T} \mathbf{s}$. The spherical constraint is invariant:

$$
\sum_{i=1}^{N} s_{i}^{2}=\sum_{i=1}^{N} y_{i}^{2} .
$$

From now on we will work in the base which diagonalizes M. The eigenvalues $\lambda_{i}$ can be readily computed:

$$
\begin{gathered}
\lambda_{1}=N-1, \\
\lambda_{k}=-1, \quad k=2, \ldots, N .
\end{gathered}
$$

The matrix $\mathbf{M}$ possesses one single positive eigenvalue and $N-1$ negative degenerate ones. In the base $\left\{y_{i}\right\}$ the energy function can be written

$$
V=-\frac{J(N-1)}{2 N} y_{1}^{2}+\frac{J}{2 N} \sum_{i \geqslant 2} y_{i}^{2} .
$$

For simplicity in what follows we fix $J=1$. Now the energy per particle is limited between $1 / 2 N-1 / 2 \leqslant v \leqslant 1 / 2 N$ or, in the thermodynamic limit, $-1 / 2 \leqslant v \leqslant 0$. In order to get the critical points of $V$ on the $(N-1)$-sphere we introduce a Lagrange multiplier to enforce the spherical constraint and define

$$
F=V+\mu\left(\sum_{i=1}^{N} y_{i}^{2}-N\right) .
$$

Now the critical points are given by $\partial F / \partial y_{i}=0$, which give

$$
\begin{gathered}
\left(2 \mu-\frac{N-1}{N}\right) y_{1}=0, \\
\left(2 \mu+\frac{1}{N}\right) y_{i}=0, \quad i \neq 1 .
\end{gathered}
$$

From this we have two possibilities, either $\mu=(N-1) / 2 N$, which gives two isolated minima $\left\{y_{1}= \pm \sqrt{N}, y_{i}=0, i \neq 1\right\}$, or $\mu=-1 / 2 N$, which gives $\left\{y_{1}=0, \Sigma_{i \neq 1} y_{i}^{2}=N\right\}$, corresponding to a degenerate maximum, completing the $(N-1)$-sphere. Consequently, the potential energy manifold has only two critical submanifolds corresponding to the minimum and maximum values of the function. There are no saddle points. This structure is trivial and allows us to visualize immediately the topology changes as the level $v$ is increased. This triviality is a property of the completely connected model only. In finite space dimensionality the potential energy manifold is more complex and already for $d=1$ it shows a nontrivial structure of saddle points [12].

The natural framework for analyzing the relation between critical points and topology changes in a manifold is Morse theory [14]. Because of the simplicity of the spherical model, one can make a very intuitive analysis of the topology changes in this case without resorting to Morse theory. The topology of the model is analyzed in the context of Morse theory in the Appendix.

Although we are interested in the behavior of the system for high dimensionality $N$, there is only one directionnamely, $y_{1}$-which breaks the spherical symmetry of the potential energy function and the problem can then be effectively analyzed in a two-dimensional plane spanned by $y_{1}$ and any other orthogonal direction. Without loss of generality, we will consider directly the case with $N=2$.

In Fig. 3 the evolution of the submanifolds $M_{v}$ is shown for four increasing values of $v$. The top left panel corresponds to a level $v$ below the minimum of the potential energy per particle, $v<-0.25$. In this case the manifold is empty; this is a forbidden region for the system. As the system crosses the level $v=-0.25$ a first topology change happens (top right panel). At this level two points are accessible in configuration space, the symmetric ground states of the system. Above this level the submanifold $M_{v}$ is diffeomorphic to two disconnected (hyper)disks (in high dimensions). This situation is represented by the bottom left panel in Fig. 3 . Note that in the $N=2$ case the submanifolds corresponding to a particular level set $v$ are represented by four points, while the submanifolds $M_{v}$ are the fraction of the two semicircles for which $V(q) / N \leqslant v$. No more topology changes happen in the submanifolds until the maximum value of the potential $v=0.25$ is reached and the whole circle (sphere) becomes accessible. The bottom right panel illustrates the situation for a level $v$ slightly below the maximum. At $v$ $=0.25$ a new topology change happens; the two disconnected sectors of the submanifolds $M_{v}$ meet each other and com- 

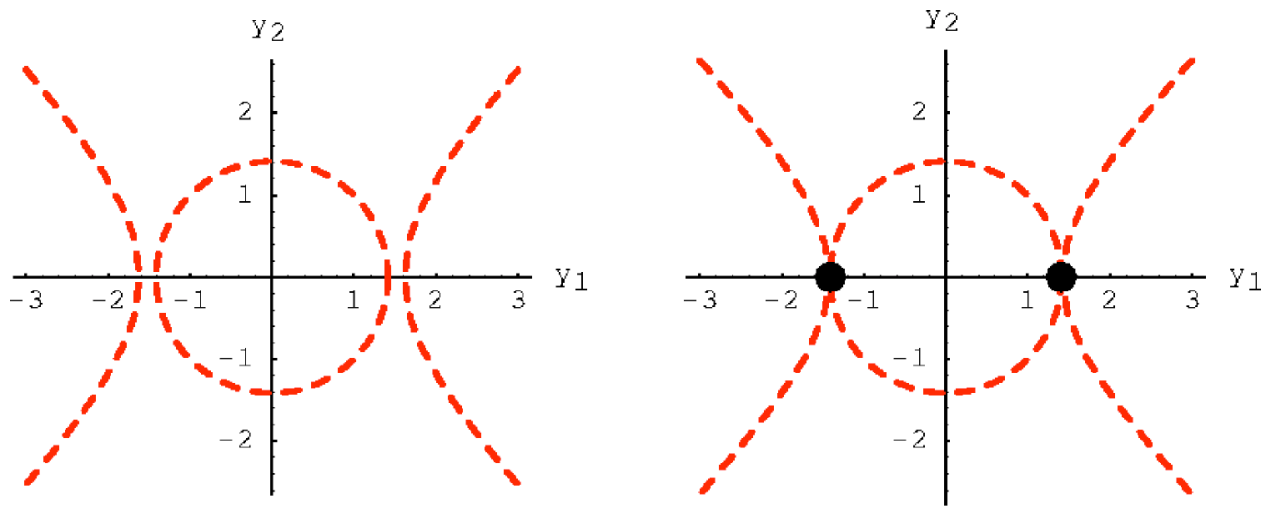

FIG. 3. (Color online) Evolution of the potential energy manifold of the $N=2$ spherical model for four levels $v=V / N$. The colored dashed lines represent the spherical constraint (a circle for $N=2$ ) and a particular level set of the function $v$. The real submanifolds depend on the level $v$ and are the continuous black sectors.
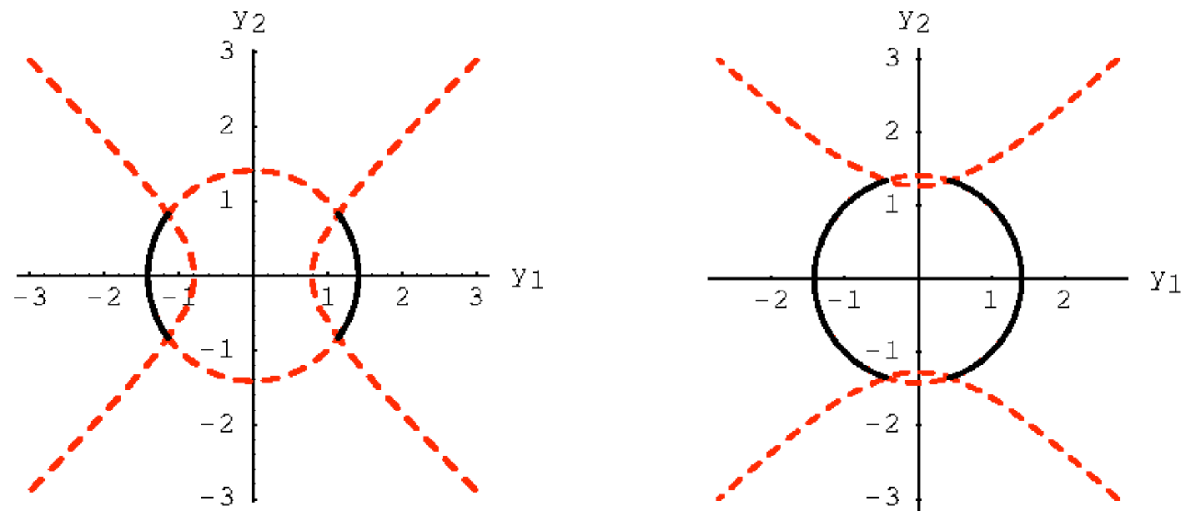

Top left: $v=-0.325$, the manifold is empty. Top right: $v=-0.25$, the manifold emerges at the two black dots (ground states). Bottom left: $v=-0.075$, two symmetric sectors of the sphere are accessible. Bottom right: $v=0.2$, near the transition the manifold is nearly completed and a large fraction of the sphere is accessible.

plete the manifold, which, for the $N$ particles system, is the $(N-1)$-sphere. The maximum of the potential energy per particle, $v_{c}=1 / 2 N$, tends to zero in the thermodynamic limit and coincides with the mean potential energy at the phase transition described in Eq. (3) and Fig. 1. This shows that the phase transition takes place at the level $v_{c}$ where a topology change in the potential energy submanifolds happens. This is what is expected according to the topological hypothesis. The evolution in the topology of the submanifolds $M_{v}$ as the level $v$ grows also illustrates in a nice way how the different sectors of the manifold $M$ become gradually accessible to the physical system. From the ground states, the only accessible at zero temperature, two symmetric regions of the (hyper)sphere become gradually accessible in accordance with the symmetry breaking nature of the phase transition in this model. In the thermodynamic limit the two regions remain disconnected until the phase transition at $v_{c}$, where the (hyper)sphere is completed, the two hemispheres connected, and the whole configuration space manifold becomes accessible to the system.

This is a simple and completely intuitive example of the topological hypothesis at work. Nevertheless, although it was already expected that a topology change must take place in correspondence with a phase transition [9], a yet open question regards the kind of topology change that might imply a phase transition. The example of the completely connected spherical model is again useful in this respect. At variance with what was observed in previously studied models, in this case it is clear that the topology change at the transition is not a strong one, at least as quantified by the change in the Euler characteristic, which is calculated in the Appendix.
There we show that the Euler characteristic is a constant equal to 2 for $1 / 2 N-1 / 2 \leqslant v<1 / 2 N$ and jumps to zero at $v_{c}=1 / 2 N$ when $N$ is even or does not change at all for $N$ odd. Clearly, from the point of view of the behavior of the Euler characteristic the change in topology is not a strong one. In the next section we consider the model in the presence of an external field $H$ which destroys the phase transition and analyze the consequences in the topology of the configuration space.

\section{B. Finite external field}

When $H \neq 0$ the energy function in the diagonal basis can be written

$$
V=-\frac{J(N-1)}{2 N} y_{1}^{2}-\sqrt{N} H y_{1}+\frac{J}{2 N} \sum_{i \geqslant 2} y_{i}^{2} .
$$

The extrema of this function evaluated on the (hyper)sphere are the same extrema of $F=V+\mu\left(\sum_{i=1}^{N} y_{i}^{2}-N\right)$. They are given by the solutions of

$$
\begin{aligned}
& \left(2 \mu-\frac{N-1}{N}\right) y_{1}=H \sqrt{N}, \\
& \left(2 \mu+\frac{1}{N}\right) y_{i}=0, \quad i \neq 1 .
\end{aligned}
$$

There are two possibilities as in the zero-field case: either $\mu=-1 / 2 N$ or $\mu \neq-1 / 2 N$. In the first case the solution is $\left\{y_{1}=-H \sqrt{N}, \sum_{i \geqslant 2} y_{i}^{2}=N\left(1-H^{2}\right)\right\}$. In the second case we obtain 

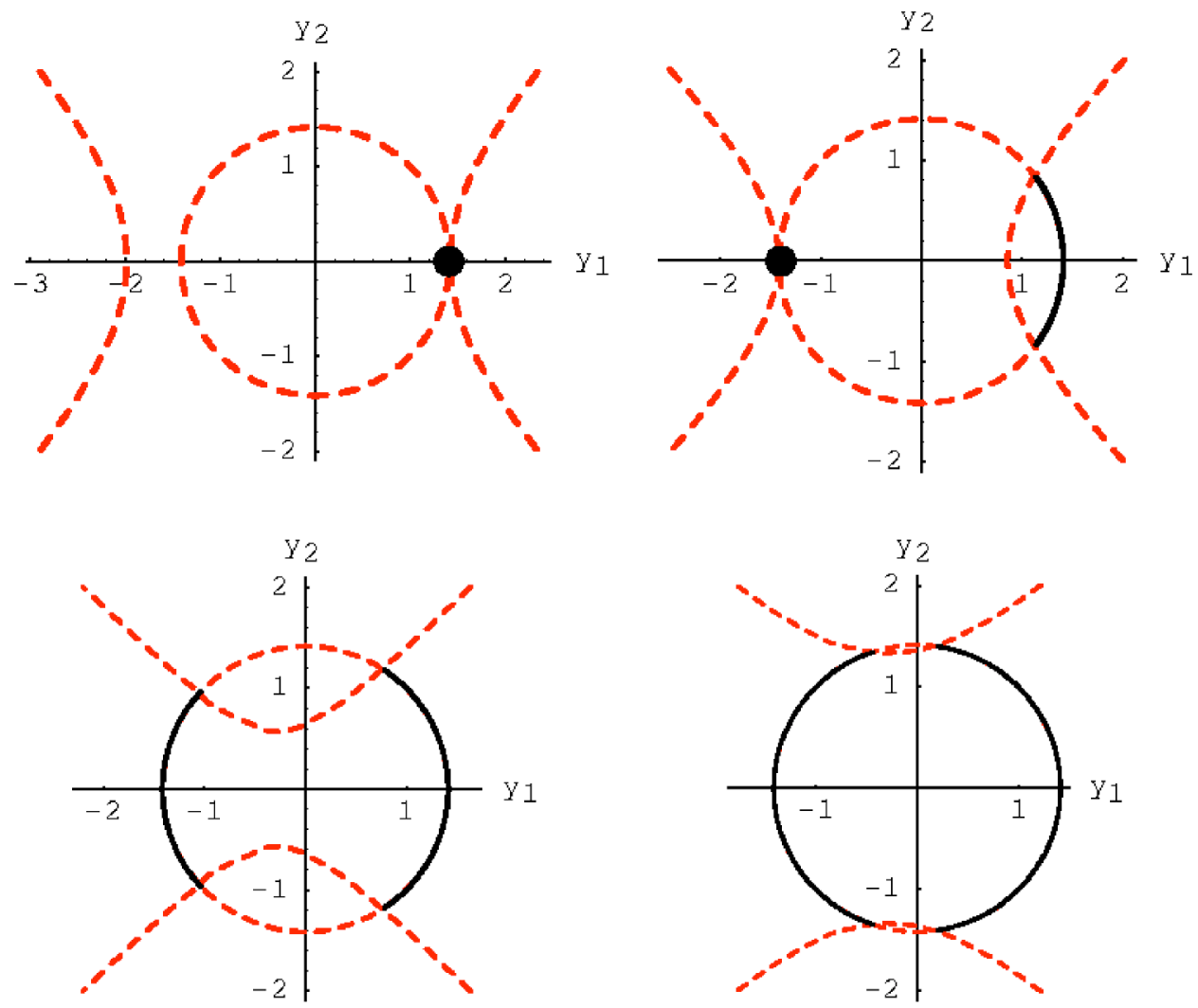

FIG. 4. (Color online) Evolution of the potential energy manifold of the $N=2$ spherical model for four levels $v$ and an external field $H=0.1$. The colored dashed lines represent a particular level set of the function $v$ and the spherical constraint (the circle). The real submanifolds are the continuous black sectors. Top left: the ground state emerges (unique black dot). Top right: a second local minimum emerges while the accessible submanifold is the continuous black arc at the right of the sphere (circle). Bottom left: at a still higher level $v$ the submanifold consists of two disconnected arcs. Bottom right: the highest thermodynamically accessible level $v=0.25$. Note that the sphere is not fully accessible. $\left\{y_{1}= \pm \sqrt{N}, y_{i}=0 \forall i \geqslant 2\right\}$. Similarly to the case $H=0$ there are two minima and a continuously degenerate maximum. The two minima now correspond to a single absolute minimum $\left\{y_{1}=\sqrt{N}, y_{i}=0 \forall i \geqslant 2\right\}$ and to a local minimum $\left\{y_{1}=-\sqrt{N}, y_{i}\right.$ $=0 \forall i \geqslant 2\}$. The corresponding energies per particle are $v_{1}$ $=-(N-1) / 2 N-H$ and $v_{2}=-(N-1) / 2 N+H$, respectively. The other critical point is in fact a critical manifold at the maximum of the energy given by $v_{3}=H^{2} / 2+1 / 2 N$. In the thermodynamic limit the potential energy per particle is $H^{2} / 2$, a level that is never reached by the system, as shown in Sec. II B.

In Fig. 4 it is shown the evolution of the submanifolds $M_{v}$ for four increasing values of $v$. One immediately recognizes the asymmetry introduced by the external field, which in these figures is $H=0.1$. The top left panel shows the level where the potential energy manifold emerges, corresponding to the ground state, which in this case is unique. At $v=H$ $-1 / 4$ the second minimum touches the sphere. This is shown in the top right panel. As the potential energy grows two disconnected regions are present, while only one of them is accessible dynamically in the thermodynamic limit. In the bottom right panel it is shown the situation at the maximum thermodynamically accessible level. The energy per particle of the maximum for $N=2$ is $v_{\max }=H^{2} / 2+1 / 4$ and goes to $H^{2} / 2$ in the thermodynamic limit. Nevertheless, the results from the thermodynamics of Sec. II B predict that the energy per particle reaches a maximum at infinite temperature which is zero, as shown in Fig. 2. Consequently the closing of the sphere is never reached by the system; the field introduces a gap $\Delta v=H^{2} / 2$ in the energy per particle that the system can never cross. The situation at the highest physically accessible level is represented in the bottom right panel of Fig. 4. One is led to the conclusion that the only topology changes in the presence of a finite external field are at the levels where the minima appear and that no other topology change takes place at higher levels of $v$, provided one restricts the analysis to the physically accessible region of the potential energy manifold. This is in agreement with the absence of a phase transition in this case: no topology change $\Rightarrow$ no phase transition. Nevertheless, this reading of the results is biased by our a priori knowledge of the thermodynamics of the system. In case the thermodynamics would not be known one could be led to the wrong conclusion that a phase transition might take place in correspondence with the maximum level of the potential energy manifold, where a topology change certainly happens. This suggests that topology alone is not enough in order to conclude if a phase transition will or will not take place in a particular system.

\section{CONCLUSIONS}

The simplicity of the completely connected spherical model allows a critical analysis of some important open questions regarding the validity of the topological hypothesis. Due to its high level of symmetry, it is possible in this model to intuitively follow the relation between the topology of the accessible manifold at any given energy level and its physical or thermodynamic behavior. In particular, the relation between the topology and the symmetry breaking transition in zero field is nicely illustrated: the phase transition takes place in the thermodynamic limit, at the level where the whole manifold, the hypersphere, becomes accessible. At this level a simple topology change takes place: the completion of the hypersphere. While this is in agreement with a 
recently proved theorem which asserts the necessity of a topology change in order for a system to have a phase transition, in this case the change is very small at variance with results from other previously studied models. Small topology changes can take place in general with no correlation with a phase transition. This is observed for example in the onedimensional $X Y$ model [4].

One can also draw some conclusions regarding the behavior of the model in the presence of an external field. In this case the comparison between thermodynamics and topology of the potential energy manifold can shed some light on the typical behavior of systems in a field. From a topological point of view few changes occur in the structure and evolution of the submanifolds $M_{v}$. For $H \leqslant J$ the degeneracy between the two minima is broken in a single ground state and a single local minimum, and a third topology change happens when the hypersphere is closed at the highest energy level. From this behavior one should be tempted to predict a phase transition similar to that in the zero-field case. Nevertheless, thermodynamics tells clearly that this is not the case; there is no phase transition in the presence of a field and the mean potential energy does not reach the top level of the potential energy manifold even at infinite temperature, a gap proportional to the square of the field amplitude existing. Consequently, to correctly read the information it is necessary to go through thermodynamics. It seems unlikely that knowledge of the topology of the potential energy manifold alone is enough in order to predict the existence of a phase transition in a generic many body system. The results presented in this work suggest that more information, coming from dynamics, is needed. Results from other models are clearly needed in order to settle this fundamental question [15]. Perhaps the strongest result predicting dynamical behavior exclusively from a static property is the celebrated Adam-Gibbs relation between relaxation time scales and configurational entropy in glasses [13]. It predicts a divergence of relaxation times when the configurational entropy $S_{c}$ associated with the number of minima of the potential energy function goes to zero. To our knowledge this prediction has never been obtained from first principles and even quantitative comparison with experiments and simulations is not conclusive.

\section{ACKNOWLEDGMENTS}

We would like to thank Lapo Casetti for useful comments and suggestions. This work was partly supported by CNPq, Brazil.

\section{APPENDIX}

In this appendix we analyze the topological evolution of the accessible manifold as the potential energy of the system is being increased from its minimum. The complete manifold $M$ for the system is the $(N-1)$-sphere. The function we define on it is $V$, the potential energy function. For a given value of the potential energy per particle $v=V / N$, the submanifold of accessible configurations is given by $M_{v}=\{y$ $\in M \mid V(y) / N \leqslant v\}$. We thus analyze the behavior of the Euler characteristic for the submanifolds $M_{v}$ for each $v, \chi\left(M_{v}\right)$, which is a topological invariant [14]. It is defined as

$$
\chi\left(M_{v}\right)=\sum_{i=0}^{N-1}(-1)^{i} b_{i}
$$

where $b_{i}$ are the Betti numbers or the number of $i$-handles that compose the manifold $M_{v}$. According to Morse theory, there is a connection between the topological transitions in a manifold and the critical points of a function defined on it. One of the results of Morse theory concerns $\chi\left(M_{v}\right)$ and is expressed by

$$
\chi\left(M_{v}\right)=\sum_{i=0}^{N-1}(-1)^{i} b_{i}=M_{-1}(V) .
$$

In case $V$ has only isolated critical points $M_{-1}(V)$ is given by

$$
M_{-1}(V)=\sum_{i=0}^{N-1}(-1)^{i} m_{i},
$$

with $m_{i}$ being the number of critical points of $V$ with index $i$ that belong to $M_{v}$. The index of a critical point is the number of negative eigenvalues of the Hessian $\mathcal{H}$ of the function at this point. In order to investigate the critical points of $V\left(y_{1}, \ldots, y_{N}\right)$ constrained to the manifold $M$, we make the analysis on the transform $F$ defined in Eq. (12). In what follows we will analyze the cases $H=0$ and $H \neq 0$.

\section{Zero external field}

As we have seen for $H=0$ the function $F$ possesses two critical levels. One of them gives $v=V / N=-(N-1) / 2 N$ corresponding to two isolated critical points $\left\{y_{1}= \pm \sqrt{N}, y_{i}=0, i\right.$ $\neq 1\}$. It is possible to verify that there is no real intersection between the manifold $M$ and the (hyper)surfaces of constant potential energy for $v<-(N-1) / 2 N$ (see top left panel in Fig. 3). The accessible submanifold $M_{v}$ for $v<-(N-1) / 2 N$ is empty, and the Euler characteristic is then identically zero: $\chi(v<-(N-1) / 2 N)=0$. The Hessian of $V$ is diagonal in the base $\left\{y_{i}\right\}$. For the two critical points appearing at $v=-(N$ $-1) / 2 N$ the eigenvalues of $\mathcal{H}$ are given by

$$
\begin{gathered}
h_{1}=0, \\
h_{i}=1, \quad i \neq 1 .
\end{gathered}
$$

The Hessian has no negative eigenvalues. The indexes of both critical points are thus zero. The Euler characteristic is then $\chi\left(M_{v}\right)=(-1)^{0} 2=2$. From $v=-(N-1) / 2 N$ while $v<1 / 2 N$ we have no other critical levels, and thus $\chi$ must remain constant up to $v=1 / 2 N$.

At $v_{c}=1 / 2 N$ the solutions of Eqs. (13) are $\left\{y_{1}=0, \Sigma_{i=2}^{N} y_{i}^{2}\right.$ $=N\}$, an $(N-2)$-dimensional critical sphere. In fact, since we have already seen that at $v=1 / 2 N$ the manifold completes itself into the (hyper)sphere $M$, we know that $\chi\left(M_{v}\right)$ $=\chi(M)=\chi\left(\mathcal{S}^{N-1}\right), \forall v \geqslant 1 / 2 N$. The Betti numbers for the sphere are well known: an $N$-sphere is composed of a 0 -handle and of an $N$-handle. The Euler characteristic for the $(N-1)$-sphere is then 


$$
\chi\left(\mathcal{S}^{N-1}\right)= \begin{cases}2 & \text { if } N \text { odd } \\ 0 & \text { if } N \text { even. }\end{cases}
$$

Although $\chi\left(M_{v}\right)$ may not change at $v_{c}$ for odd $N$, one knows that a topology change takes place on that level. This result is not contradictory since the behavior of a single topological invariant is not always enough in order to fully characterize the topology of a manifold.

\section{Finite external field}

For $H>0$ the points $\left\{y_{1}= \pm \sqrt{N}, y_{i}=0, i \neq 1\right\}$ still are solutions. However, the point $y_{1}=+\sqrt{N}$ now corresponds to the level $v_{1}=-(N-1) / 2 N-H$ and $y_{1}=-\sqrt{N}$ to $v_{2}=-(N-1) / 2 N$ $+H$. Since there is no critical value lower than $v_{1}$, the Euler characteristic for potentials below this level is identically zero: $\chi\left(M_{v}\right)=0, \forall v<v_{1}$. At the critical point corresponding to $v=v_{1}$ the eigenvalues of the Hessian are

$$
\begin{gathered}
h_{1}=0, \\
h_{i}=1+H, \quad i \neq 1 .
\end{gathered}
$$

None of the eigenvalues is negative; thus the critical point is a minimum and has index zero. The Euler invariant is then $\chi\left(M_{v}\right)=(-1)^{0} 1=1$ for $v_{1} \leqslant v<v_{2}$. The next contribution comes from the critical point $\left\{y_{1}=-\sqrt{N}, y_{i}=0 \forall i \neq 1\right\}$ at $v$
$=v_{2}=-(N-1) / 2 N+H$. At this critical point the eigenvalues of the Hessian are

$$
\begin{gathered}
h_{1}=0, \\
h_{i}=1-H, \quad i \neq 1 .
\end{gathered}
$$

We notice that the index of this critical point will depend on $H$. For $H \leqslant 1$, this critical point has index 0 , hence being a local minimum; however, for $H>1$ the index is $N-1$, and the critical point is a maximum. At $v=v_{2}$ the Euler characteristic becomes

$$
\chi\left(M_{v}\right)= \begin{cases}2 & \text { if } H \leqslant 1, \\ 0 & \text { if } H>1 \text { and } N \text { even, } \\ 2 & \text { if } H>1 \text { and } N \text { odd. }\end{cases}
$$

There is a third solution of the critical point equations which is given by $\left\{y_{1}=-\sqrt{N} H, \sum_{i=2}^{N} y_{i}=N\left(1-H^{2}\right)\right\}$. This solution only exists for $H \leqslant 1$. The corresponding critical value of the potential energy is $v_{3}=H^{2} / 2+1 / 2 N$, which is higher than the previous ones for any $H$. We have thus two different possibilities: For $H \leqslant 1, M_{v}$ coincides with $M$ [the whole (hyper)sphere] up from $v=v_{3}$. For $H>1, M_{v}$ coincides with $M$ up from $v=v_{2}<v_{3}$. Similarly to what was done in the previous section, we can use this information and simply identify $\chi\left(M_{v}\right)$ with $\chi(M)=\chi\left(\mathcal{S}^{N-1}\right)$ for either $v \geqslant v_{3}$, or $v$ $\geqslant v_{2}$.
[1] L. Caiani, L. Casetti, C. Clementi, and M. Pettini, Phys. Rev. Lett. 79, 4361 (1997).

[2] R. Franzosi, L. Casetti, L. Spinelli, and M. Pettini, Phys. Rev. E 60, R5009 (1999).

[3] R. Franzosi, M. Pettini, and L. Spinelli, Phys. Rev. Lett. 84, 2774 (2000).

[4] L. Casetti, M. Pettini, and E. G. D. Cohen, J. Stat. Phys. 111, 1091 (2003).

[5] L. Angelani, L. Casetti, M. Pettini, G. Ruocco, and F. Zamponi, Europhys. Lett. 62, 775 (2003).

[6] P. Grinza and A. Mossa, Phys. Rev. Lett. 92, 158102 (2004).

[7] L. Casetti, M. Pettini, and E. G. D. Cohen, Phys. Rep. 337, 237 (2000)
[8] L. Casetti, E. G. D. Cohen, and M. Pettini, Phys. Rev. E 65, 036112 (2002).

[9] R. Franzosi, M. Pettini, and L. Spinelli, e-print math-ph/ 0305032.

[10] R. Franzosi and M. Pettini, Phys. Rev. Lett. 92, 060601 (2004).

[11] T. H. Berlin and M. Kac, Phys. Rev. 86, 821 (1952).

[12] A. C. R. Teixeira and D. A. Stariolo (unpublished).

[13] G. Adam and J. H. Gibbs, J. Chem. Phys. 43, 139 (1965).

[14] C. Nash and S. Sen, Topology and Geometry for Physicists (Academic Press, London, 1983).

[15] Recently, a work by M. Kastner appeared (e-print cond-mat/ 0404511) with conclusions very similar to ours. 